\title{
Structure Elucidation and Biosynthesis of Orobanchol
}

\section{OPEN ACCESS}

Edited by:

Tadao Asami,

The University of Tokyo, Japan

Reviewed by: Mikihisa Umehara,

Toyo University, Japan

*Correspondence:

Yukihiro Sugimoto

yukihiro@kobe-u.ac.jp

tORCID:

Takatoshi Wakabayashi orcid.org/0000-0001-7065-6839

Kotomi Ueno

orcid.org/0000-0002-3227-2073

Yukihiro Sugimoto orcid.org/0000-0002-8767-9671

Specialty section:

This article was submitted to Plant Physiology,

a section of the journal

Frontiers in Plant Science

Received: 14 December 2021

Accepted: 18 January 2022

Published: 09 February 2022

Citation:

Wakabayashi T, Ueno $K$ and Sugimoto $Y$ (2022) Structure Elucidation and Biosynthesis of Orobanchol.

Front. Plant Sci. 13:835160. doi: 10.3389/fpls.2022.835160

\author{
Takatoshi Wakabayashi ${ }^{1+}$, Kotomi Ueno ${ }^{2+}$ and Yukihiro Sugimoto ${ }^{1 *+}$ \\ ${ }^{1}$ Graduate School of Agricultural Science, Kobe University, Kobe, Japan, ${ }^{2}$ Faculty of Agriculture, Tottori University, Tottori, \\ Japan
}

Strigolactones (SLS), a class of phytohormones that regulate diverse developmental processes, were initially characterized as host-derived germination stimulants for seeds belonging to the genera Striga, Orobanche, and Phelipanche. Orobanchol (1), which is detected in the root exudates of several plants and recognized as a prevalent SL, was first isolated from the root exudates of red clover as a germination stimulant for Orobanche minor in 1998. However, the structure of this stimulant proposed at that time was disputable considering its predicted germination-inducing activity for Striga gesnerioides. The genuine structure of orobanchol was elucidated following a decadelong controversy, which ultimately facilitated the understanding of the importance of SL stereochemistry in Striga seed germination. Recently, studies focusing on clarifying the biosynthesis pathway of orobanchol are being conducted. Cytochrome P450 monooxygenases are involved in orobanchol biosynthesis downstream of carlactonoic acid (CLA) via two pathways: either through 4-deoxyorobanchol or direct conversion from CLA. Substantial progress in the identification of more SL structures and clarification of their biosynthetic mechanisms will further contribute in the comprehension of their structural diversity's functional importance and agricultural applications. Herein, we have reviewed the history leading to the discovery of the genuine structure of orobanchol and the current understanding of its biosynthetic mechanisms.

Keywords: cytochrome P450 monooxygenase, germination, root parasitic weeds, stereochemistry, strigolactone

\section{INTRODUCTION}

Strigolactones (SLs) were initially characterized as germination stimulants for seeds belonging to the genera Striga, Orobanche, and Phelipanche, which are a renowned group of root parasitic weeds of global economic importance (Parker, 2009). Strigol (2), the first canonical SL structurally defined, was isolated from the root exudates of cotton (Gossypium hirsutum) (Cook et al., 1966, 1972). Following the isolation of strigol, the SLs sorgolactone (3) (Hauck et al., 1992), alectrol (4) (Müller et al., 1992), and orobanchol (1) (Yokota et al., 1998) were isolated from the root exudates of sorghum (Sorghum bicolor), cowpea (Vigna unguiculata), and red clover (Trifolium pratense), respectively. Consequent studies revealed that SLs not only promoted hyphal branching of arbuscular mycorrhizal fungi (Akiyama et al., 2005) but also represented a new class of 
phytohormones that regulated plant architecture (GomezRoldan et al., 2008; Umehara et al., 2008). Structurally, canonical SLs consist of tricyclic lactone (ABC ring) and butenolide (D ring) connected with an enol ether bridge (Figure 1). The structures of strigol (2) and sorgolactone (3) were unambiguously determined by X-ray crystallographic analysis and organic synthesis (Brooks et al., 1985; Sugimoto et al., 1998), whereas the genuine structures of orobanchol (1) and alectrol (4) were eventually established in 2011 (Ueno et al., 2011b). Orobanchol has been detected in the root exudates of numerous plants, including Fabaceae, Solanaceae, a few Gymnosperm species, and rice (Oryza sativa) (Xie, 2016; Wang and Bouwmeester, 2018). Several derivatives of orobanchol, such as its acetate, orobanchyl acetate (alectrol), fabacol that contains an epoxide group, and solanacol that has an aromatic A-ring, have also been identified (Müller et al., 1992; Xie et al., 2007, 2009). The illustration of the genuine structure of orobanchol allowed canonical SLs to be divided into two subgroups that were categorized in terms of their C-ring configuration, the orobanchol- and strigol-types. The C-ring configuration was found to be essential in fulfilling the structural requirements of the canonical SLs for inducing germination in Striga gesnerioides seeds (Ueno et al., 2011a; Nomura et al., 2013). The classification of the canonical SLs into the two subgroups presented an avenue to study the enzymes involved in their biosynthesis from the common intermediate, carlactonoic acid (CLA) (Zhang et al., 2014; Wakabayashi et al., 2019, 2020; Mori et al., 2020).

This review outlines the course of determining the genuine structure of orobanchol, its biological importance as a seed germination stimulant for the genus Striga, and its biosynthesis pathway at biochemical and molecular levels. The components involved in the biosynthesis of orobanchol and its related canonical SLs that are yet to be clarified are also discussed.

\section{STRUCTURE AND \\ GERMINATION-INDUCING ACTIVITY}

\section{History Leading to the Determination of the Genuine Structure of Orobanchol}

Orobanchol was isolated from the root exudates of red clover as the first germination stimulant for Orobanche minor, together with alectrol (Yokota et al., 1998). Alectrol had been previously isolated from the root exudates of cowpea as an isomer of strigol and a germination stimulant for Alectra vogelii and S. gesnerioides. A structure for alectrol was proposed based on a detailed comparison of its spectroscopic data with those of strigol (Müller et al., 1992). Since the isolated amount of orobanchol from red clover was constrained, it was considered to be a strigol-related compound and no specific structure for it was proposed. Following these reports, a series of strigol analogs, including the tentative structures of orobanchol and alectrol, were synthesized (Matsui et al., 1999a,b). The structure 5 was assigned to orobanchol by comparing its ${ }^{1} \mathrm{H}$ NMR spectra and chromatographic behavior in gas chromatographymass spectrometry, in which the C-ring configuration was consistent with that of strigol (2) (Figure 1). Chiroptical data were not utilized in the structural determination process. After about a decade, alectrol was independently re-isolated from the root exudates of red clover and cowpea (Matsuura et al., 2008; Xie et al., 2008), and its structure was reported as an acetylated product of synthetic orobanchol (6). However, synthetic orobanchol (5) and its acetate (6) did not induce seed germination in S. gesnerioides (Ueno et al., 2011a), indicating that the assigned structures of these SLs were controversial. These results triggered the re-isolation of the germination stimulants of $S$. gesnerioides from the root exudates of cowpea and red clover (Ueno et al., 2011b). The details of the bioassayguided re-isolation and unambiguous structural elucidation of these stimulants have been described in a previous review (Ueno et al., 2015). In brief, two stimulants were isolated from both cowpea and red clover root exudates. The ${ }^{1} \mathrm{H}$ NMR spectra of these stimulants suggested that they were canonical SLs having an oxygen functional group at C-4 in the B-ring. The chromatographic behavior of the stimulants in liquid chromatography-tandem mass spectrometry (LC-MS/MS) analysis was inconsistent with that of synthetic orobanchol (5) and its acetate (6) but consistent with their respective $2^{\prime}$-epimers. Additionally, the circular dichroism spectra of the stimulants were vertically inverted compared with the $2^{\prime}$-epimers of 5 and 6. Therefore, the absolute structures of orobanchol and alectrol were determined to be $\mathbf{1}$ and $\mathbf{4}$, respectively (Figure 1).

Figure 1 (1) illustrates the genuine structure of orobanchol, which has the $\left(3 \mathrm{a} R, 8 \mathrm{~b} R, 2^{\prime} R\right)$-configuration. Contrary to strigol (2), orobanchol (1) demonstrates an inverted BCjunction configuration. Dehydroxylated orobanchol and strigol, 4-deoxyorobanchol (4DO) (7) and 5-deoxystrigol (5DS) (8), respectively, have opposite $\mathrm{C}$-ring configurations, and hence, $5 \mathrm{DS}$ is also known as ent-2'-epi-4DO. It was predictable that the absolute skeletal configuration of redefined orobanchol was the ent-2'-epi-form of the strigol skeleton. Before the structural revision, 2'-epi-5-deoxystrigol (epi-5DS) had been found in the hydroponic culture media of rice seedlings (cv. Shiokari) by LC-MS/MS analysis using a reversed-phase octadecyl silica (ODS) column (Umehara et al., 2008). The detected "epi5DS" is presumed to be $4 \mathrm{DO}$ (ent-2'-epi-5DS, 7), since an LC-MS/MS analysis with an ODS column only distinguishes between diastereomers. Subsequently, it was reported that rice produces orobanchol in addition to epi-5DS (Jamil et al., 2011). Moreover, the absolute configuration of fabacyl acetate isolated from pea (Pisum sativum) was the same as that of $4 \mathrm{DO}$ (Xie et al., 2009). Therefore, the correction of the absolute configuration of orobanchol was readily accepted by the community of SL researchers.

\section{Importance of the Stereochemistry of Orobanchol in Inducing Seed Germination}

The structure of both synthetic (5) and naturally occurring orobanchol (1) have the $R$-configuration at $\mathrm{C}-2^{\prime}$, which is an 


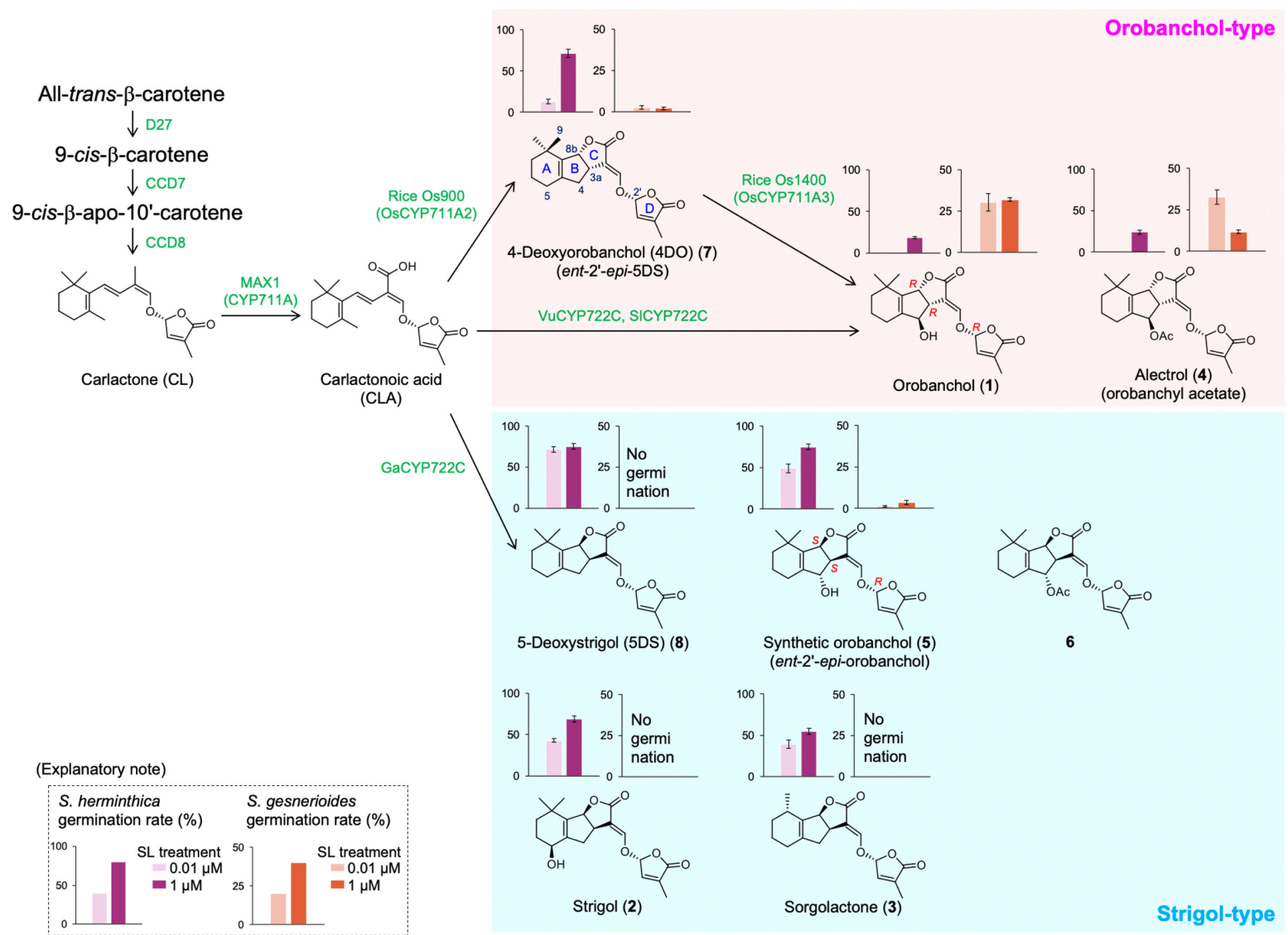

FIGURE 1 | Proposed strigolactone (SL) biosynthesis pathway from $\beta$-carotene and the seed germination induction of Striga by each SL. The sequential reactions catalyzed by D27, CCD7, and CCD8 enzymes produce carlactone (CL) from all-trans- $\beta$-carotene. CL is further converted to carlactonoic acid (CLA) by the CYP711A subfamily. Downstream of CLA, Os900/OsCYP711A2 in rice (Oryza sativa), VuCYP722C and SICYP722C in cowpea (Vigna unguiculata) and tomato (Solanum lycopersicum), respectively, and GaCYP722C in cotton (Gossypium arboreum) can produce 4-deoxyorobanchol, orobanchol, and 5-deoxystrigol, respectively. The bar graphs at the top of each SL structure indicate the germination rates of S. hermonthica and S. gesnerioides induced by each SL as reported previously (Ueno et al., 2011b; Nomura et al., 2013).

important structural feature for shoot branching inhibitory activity. Synthetic (5) as well as naturally occurring orobanchol (1) has shown to inhibit shoot branching in rice (Umehara et al., 2015). In contrast, during the structural examination of orobanchol, the importance of its stereochemistry in inducing seed germination in S. gesnerioides was suggested (Ueno et al., 2011a; Nomura et al., 2013; Figure 1). Detailed structure-activity relationship studies on 36 SL stereoisomers, including naturally occurring and synthetic ones, exemplified the strict structural requirements of the canonical SLs for inducing germination in S. gesnerioides seeds. Only a limited number of compounds, including orobanchol, induced significant germination in S. gesnerioides seeds. The SLs with high germination-inducing activity for $S$. gesnerioides seeds have a consistent C-ring configuration with that of orobanchol (1) and a hydroxy group at $\mathrm{C}-4$ with $\beta$-orientation or at C-9, the trans methyl group against the C-ring. Notably, these germination inducers of $S$. gesnerioides induced a lower germination rate in S. hermonthica, which had a more sensitive response to synthetic orobanchol (5) that has the same configuration as strigol (1). Sorghum, one of the host plants of S. hermonthica, exudes sorgomol, which also has the same configuration as strigol (2). Additionally, SLs with the same C-ring configuration as strigol suppressed the orobancholinduced germination of $S$. gesnerioides seeds. Therefore, root parasitic weeds may have evolved to germinate closer to the roots of compatible host plants where they can parasitize by strictly recognizing the configuration of the SLs. These findings indicated that not only the total amount but also the composition of SLs exuded by the host plants influence the adverse effects caused by parasitic weeds. Studies focused on elucidating the biosynthesis pathway of orobanchol were consequently pursued.

\section{BIOSYNTHESIS}

\section{Two Distinct Biosynthesis Pathways of Orobanchol}

In SL biosynthesis, D27 isomerizes all-trans- $\beta$-carotene to 9-cis$\beta$-carotene, followed by CCD7-induced cleavage to form 9-cisapo- $10^{\prime}$-carotenal, and further CCD8 catalyzed conversion to the 
SL biosynthetic precursor, carlactone (CL) (Alder et al., 2012; Seto et al., 2014; Figure 1). Cytochrome P450 monooxygenase (CYP) AtCYP711A1 encoded by MORE AXIALLY GROWTH 1 (MAX1) converts CL to CLA and is responsible for the branching phenotype observed in Arabidopsis and OsCYP711As, which belong to the same subfamily of rice, also catalyze this reaction (Abe et al., 2014; Zhang et al., 2014). Subsequently, the conversion of CL to CLA has been indicated to be a common function of the CYP711A subfamily in different plant species, suggesting that CLA is also a precursor in SL biosynthesis (Yoneyama et al., 2018). Based on the commonality of planar structure of the basic skeleton, it was assumed that the canonical SLs downstream of CLA first generated the tricyclic skeletons (5DS and 4DO), and then underwent hydroxylation and further modifications to generate strigol, orobanchol, and their acetates.

The pioneering study on canonical SL biosynthesis in japonica rice first elucidated the biosynthesis pathway of orobanchol through the conversion to 4DO (Zhang et al., 2014). The rice CYP711A subfamily shares the common functionality of CL to CLA conversion and is also involved in the conversion to orobanchol. In the rice CYP711A subfamily, OsCYP711A2/Os900 catalyzes the conversion of CL to $4 \mathrm{DO}$ via CLA, and OsCYP711A3/Os1400 catalyzes the hydroxylation of $4 \mathrm{DO}$ at C-4 to ultimately form orobanchol. Based on these results, it was assumed that the CYP711A subfamily in other plant species is also responsible for the conversion of CLA to the respective canonical SLs, including orobanchol; however, the catalyzing property of this subfamily that converts CL and CLA to canonical SLs in seed plants has been exclusively identified only in rice (Yoneyama et al., 2018). Alternatively, conventional feeding experiments observed that orobanchol producing plants (cowpea, red clover, pea, red bell pepper) that were exogenously administered with $4 \mathrm{DO}$ did not convert it to orobanchol, whereas CLA was converted to orobanchol (Iseki et al., 2018; Ueno et al., 2018). These results further suggested a direct biosynthesis pathway of orobanchol from CLA in addition to the indirect pathway through the conversion to 4DO, involving the OsCYP711A2/Os900 and OsCYP711A3/Os1400 of rice. The involvement of other enzymes besides the CYP711A subfamily in canonical SL biosynthesis has been suggested.

\section{Direct Conversion of Carlactonoic Acid to Orobanchol by CYP722C in Orobanchol Producing Plants (Cowpea and Tomato)}

Uniconazole-P, a CYP inhibitor, suppressed the conversion of CLA to orobanchol in cowpea, suggesting that CYP plays a role in this conversion. $V u C Y P 722 C$, whose function was unknown, was highlighted as a candidate gene via gene co-expression analysis using RNA-seq data of cowpea roots grown under various conditions with different SL production levels. The results of the in vitro enzyme assay conducted with a crude enzyme of recombinant VuCYP722C demonstrated that the enzyme produced orobanchol and its diastereomer, ent-2'-epi-orobanchol (5), with an opposite configuration in the C-ring, in approximately equal amounts using CLA as a substrate. Additionally, presumed 18-hydroxy-CLA was detected in the enzyme-reaction mixtures. VuCYP722C did not catalyze the conversion of $4 \mathrm{DO}$ to orobanchol, which is consistent with the previous results of the feeding experiments (Wakabayashi et al., 2019).

The enzymatic function of SlCYP722C was analyzed in tomato (Solanum lycopersicum), another representative orobanchol producer. The changes in SlCYP722C gene expressions were similar to that of known SL biosynthetic genes; upregulated under phosphate-deficient conditions that promote SL production. The recombinant enzyme exhibited an activity that was comparable to that of cowpea VuCYP722C. These results further demonstrated the existence of an alternative orobanchol biosynthesis pathway involving CYP722C (Wakabayashi et al., 2019; Figure 1).

\section{The Function of CYP722C in Tomato, a Model Orobanchol Producing Plant}

Analyses of SlCYP722C knockout tomato (SlCYP722C-KO) plants established the involvement of the CYP722C subfamily in the direct conversion of CLA to orobanchol. The root exudates of SlCYP722C-KO plants, wherein the CRISPR/Cas9 system was employed to disrupt the gene by genome editing, orobanchol and solanacol (a possible derivative of orobanchol) were demonstrated to be below-detection level using LCMS/MS analysis, and instead, CLA accumulation was observed. The modified profiles of the lacking canonical SLs were also reflected in their germination stimulation activities in the seeds of root parasitic weeds. In other words, the root exudates of SlCYP722C-KO induced significantly less germination in S. hermonthica, O. crenata, and Phelipanche aegyptiaca seeds than those of wild-type. Interestingly, the SlCYP722C-KO plants appeared similar to the wild-type plants and they did not show the prominent phenotypes of an SL-deficient mutant, such as increased shoot branching and reduced stem length (Wakabayashi et al., 2019). These observations depicted that canonical SLs were not essential for regulating shoot branching in tomato plants and further suggested that the branching inhibiting hormone was a non-canonical SL lacking the $\mathrm{ABC}$ ring structure derived from CLA, as MAX1/CYP711A mutation induces increased shoot branching (Zhang et al., 2018; Wakabayashi et al., 2019). Accordingly, canonical SLs could be more important as rhizosphere signaling molecules than shoot branching inhibitors, and preferentially secreted into the soil and facilitate plant-microbe and plantplant communications.

\section{DISCUSSION}

The determination of the genuine structures of orobanchol (1) and its acetate, orobanchyl acetate (alectrol) (4), has put an end to the long controversy regarding these structures (Ueno et al., 2011b). Orobanchol is also converted to its didehydro derivatives, didehydro-orobanchol isomers, although 




their structures and enzymes responsible for the conversion remain elusive (Zhang et al., 2018). Identification of CYP722C provided additional information on the biosynthesis pathway of orobanchol from $\beta$-carotene at a molecular level. The in vitro enzymatic reactions of VuCYP722C and SlCYP722C with CLA as a substrate yielded orobanchol and its diastereomer, ent-2'-epi-orobanchol, as products (Wakabayashi et al., 2019). These reactions further suggested that the members of the CYP722C subfamily catalyzed the two-step oxidization at the C-18 position in CLA, producing 18-oxo-CLA through 18-hydroxy-CLA. The 18-oxo-CLA then undergoes the BC ring closure reaction, without stereoselective control, to yield orobanchol isomers (Figure 2). Recently, it was reported that in a co-culture system of Escherichia coli and Saccharomyces cerevisiae co-expressing SL biosynthesis genes, orobanchol is generated by the co-expression of $V u C Y P 722 C$ with the upstream SL biosynthesis genes. However, the production of its diastereomer has not been described (Wu et al., 2021). Therefore, a more detailed functional analysis of the CYP722C subfamily is necessary. The formation of the $\mathrm{BC}$ ring without the stereoselective control of the C-ring configuration is also found in 5DS biosynthesis involving the LOW GERMINATION STIMULANT 1 (LGS1) of sorghum. It is strongly suggested that LGS1, encoding for the sulfotransferase protein, catalyzes the sulfonation of 18-hydroxy-CLA and provides an easier leaving group to afford a spontaneous non-selective BC ring formation, resulting in simultaneous production of 5DS and 4DO (Yoda et al., 2021; Figure 2). Altogether, there is likely an involvement of unknown components in the stereoselective control of the C-ring in the conversion of 18oxo-CLA to orobanchol and the sulfate ester of 18-hydroxyCLA to 5DS.

The CYP722C subfamily is widely conserved in dicot plants, regardless of the type of SL produced (orobanchol- or strigoltype). GaCYP722C of cotton ( $G$. arboreum), which generates 5DS as a strigol-type SL, catalyzes the conversion of CLA to 5DS, but it is not involved in the conversion to 4DO (Wakabayashi et al., 2020). Alternatively, GaCYP722C catalyzes stereoselective $\mathrm{BC}$ ring formation, unlike VuCYP722C and SlCYP722C. In addition, it has been reported that the CYP722Cs of birdsfoot trefoil (Lotus japonicus) and woodland strawberry (Fragaria vesca) are involved in the conversion of CLA to 5DS (Mori et al., 2020; Wu et al., 2021). The CYP722C subfamily members are the key enzymes involved in the biosynthesis of canonical SLs, regardless of their C-ring configuration. The differences in their catalytic activity may be due to the differences in the amino acid residues at the catalytic site and conformation of the protein structure domains. Structural biological approaches may clarify the mechanisms regulating the C-ring configuration in canonical SL biosynthesis.

Although much progress has been made in understanding the diverse structures of SLs and their biosynthetic mechanisms, 
the physiological significance of SL stereochemistry remains largely unexplored. If the mechanism by which plants control the stereochemistry of the C-ring to produce both types of SLs could be elucidated, it would then become possible to artificially control their structures through genetic engineering. The knowledge obtained from this approach will greatly contribute in comprehending the role of SLs. Additionally, the precise control of SL functions is predicted to have agricultural applications, such as management of root parasitic weeds and promotion of mycorrhizal symbiosis.

\section{REFERENCES}

Abe, S., Sado, A., Tanaka, K., Kisugi, T., Asami, K., Ota, S., et al. (2014). Carlactone is converted to carlactonoic acid by MAX1 in Arabidopsis and its methyl ester can directly interact with AtD14 in vitro. Proc. Natl. Acad. Sci. U S A. 111, 18084-18089. doi: 10.1073/pnas.1410801111

Akiyama, K., Matsuzaki, K., and Hayashi, H. (2005). Plant sesquiterpenes induce hyphal branching in arbuscular mycorrhizal fungi. Nature 435, 824-827. doi: 10.1038/nature03608

Alder, A., Jamil, M., Marzorati, M., Bruno, M., Vermathen, M., Bigler, P., et al. (2012). The path from $\beta$-carotene to carlactone, a strigolactone-like plant hormone. Science 335, 1348-1351. doi: 10.1126/science.1218094

Brooks, D. W., Bevinakatti, H. S., and Powell, D. R. (1985). The absolute structure of (+)-strigol. J. Org. Chem. 50, 3779-3781. doi: 10.1021/jo00220a020

Cook, C. E., Whichard, L. P., Turner, B., Wall, M. E., and Egley, G. H. (1966). Germination of witchweed (Striga lutea Lour.): Isolation and properties of a potent stimulant. Science 154, 1189-1190. doi: 10.1126/science.154.3753.1189

Cook, C. E., Whichard, L. P., Wall, M., Egley, G. H., Coggon, P., Luhan, P. A., et al. (1972). Germination stimulants. II. Structure of strigol, a potent seed germination stimulant for witchweed (Striga lutea). J. Am. Chem. Soc. 94, 6198-6199. doi: 10.1021/ja00772a048

Gomez-Roldan, V., Fermas, S., Brewer, P. B., Puech-Pagès, V., Dun, E. A., Pillot, J. P., et al. (2008). Strigolactone inhibition of shoot branching. Nature 455, 189-194. doi: 10.1038/nature07271

Hauck, C., Müller, S., and Schildknecht, H. (1992). A germination stimulant for parasitic flowering plants from Sorghum bicolor, a genuine host plant. J. Plant Physiol. 139, 474-478. doi: 10.1016/S0176-1617(11)80497-9

Iseki, M., Shida, K., Kuwabara, K., Wakabayashi, T., Mizutani, M., Takikawa, H., et al. (2018). Evidence for species-dependent biosynthetic pathways for converting carlactone to strigolactones in plants. J. Exp. Bot. 69, 2305-2318. doi: $10.1093 / \mathrm{jxb} / \mathrm{erx} 428$

Jamil, M., Charnikhova, T., Cardoso, C., Jamil, T., Ueno, K., Verstappen, F., et al. (2011). Quantification of the relationship between strigolactones and Striga hermonthica infection in rice under varying levels of nitrogen and phosphorus. Weed Res. 51, 373-385. doi: 10.1111/j.1365-3180.2011.00847.x

Matsui, J., Bando, M., Kido, M., Takeuchi, Y., and Mori, K. (1999a). Synthetic disproof of the structure proposed for alectrol, the germination stimulant from Vigna unguiculata. Eur. J. Org. Chem. 1999, 2195-2199. doi: 10.1002/(SICI) 1099-0690(199909)1999:9<2195::AID-EJOC2195<3.0.CO;2-R

Matsui, J., Yokota, T., Bando, M., Takeuchi, Y., and Mori, K. (1999b). Synthesis and structure of orobanchol, the germination stimulant for Orobanche minor. Eur. J. Org. Chem. 1999, 2201-2210. doi: 10.1002/(SICI)1099-0690(199909)1999: 9<2201::AID-EJOC2201<3.0.CO;2-Q

Matsuura, H., Ohashi, K., Sasako, H., Tagawa, N., Takano, Y., Ioka, Y., et al. (2008). Germination stimulant from root exudates of Vigna unguiculata. Plant Growth Regul. 54, 31-36. doi: 10.1007/s10725-007-9224-9

Mori, N., Nomura, T., and Akiyama, K. (2020). Identification of two oxygenase genes involved in the respective biosynthetic pathways of canonical and noncanonical strigolactones in Lotus japonicus. Planta 251:40. doi: 10.1007/s00425019-03332-x

Müller, S., Hauck, C., and Schildknecht, H. (1992). Germination stimulants produced by Vigna unguiculata Walp cv Saunders Upright. J. Plant Growth Regul. 11, 77-84. doi: 10.1007/BF00198018

\section{AUTHOR CONTRIBUTIONS}

TW, KU, and YS wrote the review. All authors contributed to the article and approved the submitted version.

\section{FUNDING}

This work was supported in part, by the JST/JICA SATREPS (JPMJSA1607 to YS), JST ACT-X (JPMJAX20BM to TW), and JSPS KAKENHI (25292065 to YS and 20K15459 to TW).

Nomura, S., Nakashima, H., Mizutani, M., Takikawa, H., and Sugimoto, Y. (2013). Structural requirements of strigolactones for germination induction and inhibition of Striga gesnerioides seeds. Plant Cell Rep. 32, 829-838. doi: 10.1007/s00299-013-1429-y

Parker, C. (2009). Observations on the current status of Orobanche and Striga problems worldwide. Pest. Manag. Sci. 65, 453-459. doi: 10.1002/ps.1713

Seto, Y., Sado, A., Asami, K., Hanada, A., Umehara, M., Akiyama, K., et al. (2014). Carlactone is an endogenous biosynthetic precursor for strigolactones. Proc. Natl. Acad. Sci. U S A. 111, 1640-1645. doi: 10.1073/pnas.1314805111

Sugimoto, Y., Wigchert, S. C. M., Thuring, J. W. J. F., and Zwanenburg, B. (1998). Synthesis of all eight stereoisomers of the germination stimulant sorgolactone. J. Org. Chem. 63, 1259-1267. doi: 10.1021/jo9718408

Ueno, K., Fujiwara, M., Nomura, S., Mizutani, M., Sasaki, M., Takikawa, H., et al. (2011a). Structural requirements of strigolactones for germination induction of Striga gesnerioides seeds. J. Agric. Food Chem. 59, 9226-9231. doi: 10.1021/ jf202418a

Ueno, K., Nakashima, H., Mizutani, M., Takikawa, H., and Sugimoto, Y. (2018). Bioconversion of 5-deoxystrigol stereoisomers to monohydroxylated strigolactones by plants. J. Pestic. Sci. 43, 198-206. doi: 10.1584/jpestics.D18021

Ueno, K., Nomura, S., Muranaka, S., Mizutani, M., Takikawa, H., and Sugimoto, Y. (2011b). Ent-2'-epi-orobanchol and its acetate, as germination stimulants for Striga gesnerioides seeds isolated from cowpea and red clover. J. Agric. Food Chem. 59, 10485-10490. doi: 10.1021/jf2024193

Ueno, K., Sugimoto, Y., and Zwanenburg, B. (2015). The genuine structure of alectrol: end of a long controversy. Phytochem. Rev. 14, 835-847. doi: 10.1007/ s11101-014-9380-2

Umehara, M., Cao, M., Akiyama, K., Akatsu, T., Seto, Y., Hanada, A., et al. (2015). Structural requirements of strigolactones for shoot branching inhibition in rice and Arabidopsis. Plant Cell Physiol. 56, 1059-1072. doi: 10.1093/pcp/ pcr028

Umehara, M., Hanada, A., Yoshida, S., Akiyama, K., Arite, T., Takeda-Kamiya, N., et al. (2008). Inhibition of shoot branching by new terpenoid plant hormones. Nature 455, 195-200. doi: 10.1038/nature07272

Wakabayashi, T., Hamana, M., Mori, A., Akiyama, R., Ueno, K., Osakabe, K., et al. (2019). Direct conversion of carlactonoic acid to orobanchol by cytochrome P450 CYP722C in strigolactone biosynthesis. Sci. Adv. 5:eaax9067. doi: 10.1126/ sciadv.aax 9067

Wakabayashi, T., Shida, K., Kitano, Y., Takikawa, H., Mizutani, M., and Sugimoto, Y. (2020). CYP722C from Gossypium arboreum catalyzes the conversion of carlactonoic acid to 5-deoxystrigol. Planta 251:97. doi: 10.1007/s00425-02003390-6

Wang, Y., and Bouwmeester, H. J. (2018). Structural diversity in the strigolactones. J. Exp. Bot. 69, 2219-2230. doi: 10.1093/jxb/ery091

Wu, S., Ma, X., Zhou, A., Valenzuela, A., Zhou, K., and Li, Y. (2021). Establishment of strigolactone-producing bacterium-yeast consortium. Sci. Adv. 7, 1-14. doi: 10.1126/sciadv.abh4048

Xie, X. (2016). Structural diversity of strigolactones and their distribution in the plant kingdom. J. Pestic. Sci. 41, 175-180. doi: 10.1584/jpestics.J16-02

Xie, X., Kusumoto, D., Takeuchi, Y., Yoneyama, K., Yamada, Y., and Yoneyama, K. (2007). 2'-Epi-orobanchol and solanacol, two unique strigolactones, germination stimulants for root parasitic weeds, produced by tobacco. J. Agric. Food Chem. 55, 8067-8072. doi: 10.1021/jf0715121 
Xie, X., Yoneyama, K., Harada, Y., Fusegi, N., Yamada, Y., Ito, S., et al. (2009). Fabacyl acetate, a germination stimulant for root parasitic plants from Pisum sativum. Phytochemistry 70, 211-215. doi: 10.1016/j.phytochem.2008.12.013

Xie, X., Yoneyama, K., Kusumoto, D., Yamada, Y., Yokota, T., Takeuchi, Y., et al. (2008). Isolation and identification of alectrol as (+)-orobanchyl acetate, a germination stimulant for root parasitic plants. Phytochemistry 69, 427-431. doi: 10.1016/j.phytochem.2007.07.017

Yoda, A., Mori, N., Akiyama, K., Kikuchi, M., Xie, X., Miura, K., et al. (2021). Strigolactone biosynthesis catalyzed by cytochrome $\mathrm{P} 450$ and sulfotransferase in sorghum. New Phytol. 232, 1999-2010. doi: 10.1111/nph.17737

Yokota, T., Sakai, H., Okuno, K., Yoneyama, K., and Takeuchi, Y. (1998). Alectrol and orobanchol, germination stimulants for Orobanche minor, from its host red clover. Phytochemistry 49, 1967-1973. doi: 10.1016/S0031-9422(98)00419-1

Yoneyama, K., Mori, N., Sato, T., Yoda, A., Xie, X., Okamoto, M., et al. (2018). Conversion of carlactone to carlactonoic acid is a conserved function of MAX1 homologs in strigolactone biosynthesis. New Phytol. 218, 1522-1533. doi: 10. 1111/nph.15055

Zhang, Y., Cheng, X., Wang, Y., Díez-Simón, C., Flokova, K., Bimbo, A., et al. (2018). The tomato MAX1 homolog, SlMAX1, is involved in the biosynthesis of tomato strigolactones from carlactone. New Phytol. 219, 297-309. doi: 10. $1111 /$ nph.15131
Zhang, Y., van Dijk, A. D. J., Scaffidi, A., Flematti, G. R., Hofmann, M., Charnikhova, T., et al. (2014). Rice cytochrome P450 MAX1 homologs catalyze distinct steps in strigolactone biosynthesis. Nat. Chem. Biol. 10, 1028-1033. doi: $10.1038 /$ nchembio. 1660

Conflict of Interest: The authors declare that the research was conducted in the absence of any commercial or financial relationships that could be construed as a potential conflict of interest.

Publisher's Note: All claims expressed in this article are solely those of the authors and do not necessarily represent those of their affiliated organizations, or those of the publisher, the editors and the reviewers. Any product that may be evaluated in this article, or claim that may be made by its manufacturer, is not guaranteed or endorsed by the publisher.

Copyright $\odot 2022$ Wakabayashi, Ueno and Sugimoto. This is an open-access article distributed under the terms of the Creative Commons Attribution License (CC BY). The use, distribution or reproduction in other forums is permitted, provided the original author(s) and the copyright owner(s) are credited and that the original publication in this journal is cited, in accordance with accepted academic practice. No use, distribution or reproduction is permitted which does not comply with these terms. 